\title{
Propiedades psicométricas del Manual para la Valoración del Riesgo de Violencia contra la Pareja (SARA) en agresores chilenos
}

\section{Psychometric properties of the Manual for the Evaluation of the Risk of Violence against the Couple (SARA) in Chilean aggressors}

\author{
René Gallardo \\ Escuela de Psicología, Universidad Austral de Chile, Chile \\ Andrés Concha-Salgado \\ Departamento de Psicología, Universidad de La Frontera, Chile
}

Rec (15 de junio 2015) Acept (10 de marzo 2017)

\begin{abstract}
Resumen
La finalidad de este estudio instrumental fue evaluar las propiedades psicométricas del Manual para la Valoración del Riesgo de Violencia contra la Pareja SARA (The Spousal Assault Risk Assessment) por medio de la adaptación y de la obtención de indicadores de validez concurrente y diagnóstica y de consistencia interna. La muestra estuvo conformada por 62 participantes, 32 agresores contra la pareja y 30 no agresores contra la pareja (muestra de contraste). Se administró el Manual SARA adaptado, junto al Eysenck Personality Questionnaire (EPQ-R) versión chilena y el Parental Bonding Instrument (PBI) versión chilena. Los resultados sugieren buenos coeficientes de consistencia interna, validez concurrente y validez diagnóstica. Se discuten las implicancias de los hallazgos.

Palabras clave: Violencia contra la pareja, Manual SARA, Validez, Confiabilidad.
\end{abstract}

\begin{abstract}
The purpose of this instrumental study was to evaluate the psychometric properties of the Spousal Assault Risk Assessment (SARA) through an adaptation process and evaluating concurrent and diagnostic validity and internal consistency. The participants were 62, 32 offenders against their couple and 30 non-offenders against their couple (comparison sample). The instruments were SARA adapted, the chilean version of the Eysenck Personality Questionnaire (EPQ-R) and the chilean version of the Parental Bonding Instrument (PBI). The results suggest good reliability coefficients, concurrent validity and diagnostic accuracy. The implications of these findings are discussed.

Keywords: Spousal Assault, SARA, Validity, Reliability.
\end{abstract}

\footnotetext{
Correspondencia:René Gallardo, Escuela de Psicología Universidad Austral de Chile, Los Pinos s/n, Balneario Pelluco, Puerto Montt, Chile. Teléfono de contacto: +(56) 065 2277153. Correo electrónico rgallardo@spm.uach.cl, andres.concha@ufrontera.cl

Nota de los Autores

Este estudio forma parte de un proyecto de investigación DID-S-2013-54 financiado por la Dirección de Investigación y Desarrollo de la Universidad Austral de Chile. Se agradece a Karen Andrades, Valentina Cerpa, Nancy Martínez, Natalia Ubilla, Miriam Ramírez, Catalina Millán, Sebastián Hernández y Ana Barría, por su valioso aporte en distintas partes del proyecto.
} 


\section{Introducción}

No cabe duda que la violencia constituye un fenómeno social de amplio impacto en nuestra realidad nacional. Un estudio realizado por el Servicio Nacional de la Mujer (SERNAM, 2010) que lleva por título "Violencia Intrafamiliar, en cifras" señala que en Chile el $35,7 \%$ de las mujeres entre 15 y 59 años han sufrido violencia alguna vez en su vida. A su vez, los datos registrados en la región de Los Lagos, se correlacionan con las cifras indicadas anteriormente. En el año 2013, Carabineros de Chile registró un total de 4.281 casos de violencia contra la pareja, en donde 3.646 la agredida había sido la mujer, frente a 635 donde el agredido había sido el hombre. A su vez la violencia que más prevaleció fue la psicológica, la cual estuvo presente en 2.113 casos.

En cuanto a su definición, la violencia es entendida como todo tipo de abuso de poder que se lleve a cabo a través de diversos mecanismos como: coacciones psíquicas, físicas, verbales, sexuales, económicas o sociales, que intenten influir, limitar o romper la voluntad de una persona (AndrésPueyo, 2008), especialmente la ejercida por el hombre sobre la mujer, constituyendo esta una de las formas más graves de violencia interpersonal y una elevada prevalencia (Andrés-Pueyo, 2008).

En este ámbito, y en un nivel más específico, la violencia contra la pareja (VCP) no es sólo un sinónimo de agresión física sobre la pareja, si no que iría más allá:

"es un patrón de conductas violentas y coercitivas que incluyen los actos de violencia física contra la pareja, pero también el maltrato y abuso psicológico las agresiones sexuales, el aislamiento y control social, el acoso sistemático y amenazante, la intimidación, la humillación, la extorsión económica y las amenazas más diversas". (Andrés-Pueyo, López y Álvarez, 2008, p.109).

Todas estas actividades, que se pueden extender en el tiempo tendrían como finalidad someter a la víctima al poder y control del agresor.

Los estudios en torno a la violencia han ahondado en las vinculaciones que esta puede tener con otras variables de carácter psicológico; tal es el caso de la dimensión de personalidad Neuroticismo y el tipo de apego que tenga el agresor.

En cuanto a la primera de ellas, y de acuerdo al modelo PEN propuesto por Eysenck (1982), existirían en la estructura de la personalidad 3 grandes rasgos, de los cuales Neuroticismo es uno de ellos. Este se refiere a características comportamentales tales como: variabilidad e inestabilidad emocional, hipersensibilidad, ansiedad y desarreglos somáticos (jaquecas, dolores de espalda, problemas digestivos). Así, un sujeto agresor tendría baja autoestima, dependencia emocional (miedo a ser abandonado) y restricción en la expresión de emociones, lo que lo llevaría a acumular tensión, que en algún momento se desborda (Ibaceta, 2004).

Por otra parte, Cortés, Mestre, Tur, Samper y Nácher (2007) con respecto a la correlación existente entre agresividad física y verbal e inestabilidad emocional, destacan en sus resultados que la "agresividad física y verbal mantiene una fuerte conexión significativa positiva y directa con la inestabilidad emocional. Por su parte, la estabilidad emocional como factor estructural de la personalidad, salvaguarda a la persona de manifestaciones agresivas, físicas o verbales, al tiempo que estimula los comportamientos de acercamiento a los demás y las respuestas prosociales" (p. 220).

De esta forma, los neuróticos (inestables emocionalmente) aumentan su probabilidad de ser agresivos ya que son inestables emocional y afectivamente, controlan mal sus afectos y emociones, tienen baja tolerancia a la frustración, son inseguros, dependientes, conflictivos consigo mismo y con un alto nivel de ansiedad flotante. Esto en gran parte debido a que en sus relaciones de pareja, se vuelven dependientes, afectiva y emocionalmente; en caso de que las mujeres deciden alejarse del sujeto, este tipo de hombres pueden presentar altos niveles de ansiedad y sentimientos de frustración a lo cual pueden reaccionar de manera desmedida, acosando a su pareja, coaccionándola, persiguiéndola y/o agrediéndola físicamente (Castellano, Lachica, Molina y Villanueva, 2004).

La segunda variable que estaría relacionada con la violencia contra la pareja es el tipo de apego, el cual, a pesar de ser formulado como una teoría referida al vínculo afectivo del bebé con su cuidador (Bowlby, 1973), es un componente de la experiencia humana presente durante toda la vida (Fraley y Shaver, 2000); manifestándose, naturalmente, en las relaciones de pareja que iniciamos y mantenemos en nuestra vida adulta. Los vínculos de apego se han clasificado como seguro, evitante y ambivalente dependiendo de la respuesta que los bebes tuvieron ante la separación y el encuentro, en el procedimiento denominado la situación extraña desarrollada por Ainsworth et al. (1978). Más tarde Solomon describió una cuarta categoría insegura denominada desorganizado. Posteriormente, Bartholomew y Horowiz (1991) desarrollaron un modelo de apego adulto el cual se compone de cuatro categorías bi-dimensionales: seguro, temeroso, preocupado y rechazante, teniendo cada 
una de estas categorías una imagen del self y de los otros específica.

Resultados en esta línea sugieren que los agresores contra sus parejas, en comparación con sujetos no violentos, tendrían un estilo de apego inseguro (rechazante y preocupado), poca estabilidad emocional y gran ansiedad frente al rechazo o abandono por parte de la pareja (Babcock et al., 2000; Holtzworth- Munroe et al., 2003). Se plantea como explicación de esta asociación el hecho de ser sujetos especialmente vulnerables a los sentimientos de abandono y con una mayor necesidad de dominio en las relaciones íntimas (Loinaz y Echeburúa, 2012). De igual modo se ha consignado que la relación entre ser maltratador y apego inseguro se ve influida también por características de personalidad tales como ansiedad de separación y desconfianza hacia la pareja (Buck, Leenaars, Emmelkamp y Van Marle, 2012).

En cuanto a la evaluación de la violencia contra la pareja, en la década del noventa se desarrollaron instrumentos de valoración de riesgo, entre ellos, el SARA (Spouse Assault Risk Assesment) desarrollado en Canadá por Kropp, Hart, Webster y Eaves (1994), el cual es una guía que permite valorar el riesgo de violencia contra la pareja, a partir del análisis de cinco dimensiones que abarcan: el pasado y el presente del agresor(a), datos a nivel personal, laboral, familiar, etc. Requiere que los examinadores evalúen sobre la presencia o ausencia de los factores de riesgo que en ella se indican, e informar a los organismos correspondientes la probabilidad de que un agresor(a) vuelva a reiterar la conducta violenta especialmente la física y/o sexual, en un tiempo aproximado entre tres y seis meses después de la aplicación (Andrés-Pueyo, 2009).

Esta herramienta busca cumplir con los siguientes objetivos: (1) tomar decisiones adecuadas en la intervención clínica, (2) aumentar el rigor y sobre todo la transparencia de las decisiones, (3) proteger a las víctimas y sus bienes, (4) gestión de la seguridad de las víctimas en base los riesgos previstos y (5) prevenir la violencia (Andrés-Pueyo, 2009).

El SARA original en su versión canadiense, surge debido a la falta de una herramienta adecuada para medir el riesgo de violencia contra la pareja, por lo que diversos autores desarrollaron posteriormente un conjunto de directrices profesionales, que denominaron Guía de Evaluación de Riesgo de Violencia contra la pareja (SARA) (Kropp et al., 1994, 1995, 1998).

En relación con la versión original, la evaluación de las propiedades psicométricas, se realizó mediante las aplicaciones a seis muestras de delincuentes adultos varones (Kropp y Hart, 2000). En general, los resultados dan cuenta de un alto nivel de confiabilidad inter juez (coeficiente de correlación intraclase), específicamente las que conciernen a los factores de riesgo individuales y al riesgo total percibido. Además, según los análisis estructurales realizados a los factores de riesgo, estos indicaron niveles de consistencia interna y homogeneidad de ítems moderada. Así también, en una de las muestras, el SARA fue capaz de discriminar significativamente entre los ofensores con y sin un historial de violencia contra la pareja y en otra, entre ofensores contra la pareja reincidente y no reincidente. Finalmente, los puntajes del SARA mostraron buena validez convergente y discriminante en comparación a los otros instrumentos de medición utilizados relacionados a criminalidad violenta y violencia en general (Kropp y Hart, 2000).

Por su parte en el 2005, Andrés-Pueyo y López llevaron a cabo una adaptación española de este instrumento, basándose en la segunda versión del SARA canadiense (Kropp et al., 1995). Los instrumentos utilizados en esta investigación, fueron el SARA y el Protocolo de recogida de información. La muestra estuvo compuesta por 102 parejas que se encontraban separadas en el momento de la investigación y 204 sujetos, de los cuales se hizo un seguimiento de 12 meses para evaluar la reincidencia de los agresores.

Algunos resultados de este estudio, guardan relación con que se encontraron los mismos factores de riesgo que en el estudio de Kroppp y Hart (2000) entre ellos, presencia de antecedentes familiares de conductas violentas en la infancia, tanto en la víctima como en el agresor. En la misma línea, en los agresores se identificó como factor de riesgo: dificultades de aprendizaje y trastornos de conducta en la infancia, ira, hostilidad o irritabilidad, inestabilidad emocional, habían agredido a otras personas y tenían otros antecedentes delictivos. Además, presentaban minimización extrema o negación de la violencia, con un incremento de la frecuencia y/o la gravedad de las agresiones (López y Andrés-Pueyo, 2007).

Actualmente, en Chile se utiliza el SARA versión española, sin embargo, hasta la revisión bibliográfica realizada para este trabajo, no se encontraron publicaciones de estudios psicométricos de este instrumento en Chile, es por ello que se considera necesario adaptar y evaluar sus propiedades psicométricas, por ello se propuso como objetivo general: evaluar las propiedades psicométricas del SARA en una muestra de agresores contra la pareja y no agresores contra la pareja del sur de Chile. Del mismo modo se formularon cuatro objetivos específicos: (1) adaptar los ítems del instrumento; (2) evaluar la confiabilidad del instrumento; (3) evaluar la validez concurrente del instrumento, y (4) evaluar la validez diagnóstica del instrumento. 
Si bien, este es un estudio instrumental, de igual forma se propusieron algunas hipótesis a partir de la relación esperada del constructo Violencia contra la pareja con otras variables criterio como son el Neuroticismo, el tipo de muestra (agresores y no-agresores contra la pareja) y el tipo de Apego (seguro e inseguro). Así, las hipótesis planteadas son: (H1) existirá correlación significativa y directa entre los puntajes de Neuroticismo y los puntajes del SARA; (H2) existirán diferencias significativas en los puntajes del SARA según el tipo de muestra; (H3) existirán diferencias significativas en los puntajes del SARA según el tipo de apego.

\section{Método}

El diseño de esta investigación, según Montero y León (2007) puede clasificarse como un estudio instrumental. Se utilizaron los criterios para el desarrollo de estudios instrumentales de Carretero-Dios y Pérez (2005) y Muñiz, Elousa y Hambleton (2013) para asegurar la adaptación del instrumento a la realidad cultural chilena.

\section{Participantes}

Se trabajó con una muestra no probabilística intencionada. La muestra del estudio estuvo conformada en su totalidad por hombres, todos mayores de dieciocho años, contactados a través de Gendarmería de Chile de la ciudad de Puerto Montt, tanto del sistema abierto como del sistema cerrado. Los dos grupos de estudio (agresores contra la pareja y no-agresores contra la pareja) son el resultado de la clasificación de los participantes en base al historial de violencia previo que poseían.

La muestra de agresores contra la pareja estuvo conformada por 32 sujetos (de un universo de 87 ), para este grupo los criterios de inclusión fueron: (a) haber mantenido al menos una relación sentimental con otra persona, (b) que se encontraran condenados por delitos relacionados a violencia intrafamiliar, específicamente violencia contra la pareja y (c) que poseyeran causas anteriores asociadas a dicho delito. Es decir, se incluyen a sujetos con antecedentes de violencia intrafamiliar (agresión contra la pareja).

Por otro lado, la muestra de contraste, conformada por no agresores contra la pareja, fue de 30 sujetos (de un universo de 40). En este grupo los criterios de inclusión fueron: (a) haber mantenido al menos una relación sentimental con otra persona, (b) no tener antecedentes de violencia contra la pareja en el sistema penal. En síntesis, en este grupo, lo sujetos poseían antecedentes penales, pero no de violencia intrafamiliar (agresión contra sus parejas). Las principales características de la muestra se resumen en la Tabla 1.

\section{Instrumentos}

Las variables en este estudio fueron: violencia contra la pareja, Neuroticismo y tipo de apego.

La primera de ellas fue medida a través del Manual para la valoración del riesgo de violencia contra la pareja (SARA). Este instrumento posee 20 ítems agrupados en 4 dimensiones (Historial delictivo, Variables psicosociales, Historial de violencia contra la pareja y Delito/Agresión actual), la forma de calificar cada ítem es de 0 a 2, por ejemplo; en el ítem 1 Violencia anterior contra familiares, el evaluador puede asignar un 0 si "no existe evidencia", un 1 si "hay amenaza de agresión" y un 2 si "hay agresión física o intento real de agresión” (ver Anexo 1).

La segunda variable, Neuroticismo, se midió con el Cuestionario de Personalidad de Eysenck (EPQ-R) versión chilena, adaptada para población chilena por Bustos y Meneses (1991) y estandarizada por Kaplan y Liberman (1992). El test se puede aplicar de manera individual como colectiva, consta de 100 ítems los cuales se dividen en cuatro dimensiones, siendo Estabilidad-Neuroticismo (N) la que se utilizó en el análisis. Ésta posee 24 ítems, y evalúa si el sujeto es estable o neurótico (Gempp y Chesta, 2007).

Por último, el tipo de apego se determinó y midieron a partir de los estilo parentales, los cuales se medirán a través del Parental Bonding Instrument (PBI) versión chilena (Dávila, Ormeño y Vera, 1998) estandarizada en población desde los 16 hasta los 64 años de edad, de ambos sexos. Dicho cuestionario posee normas según sexo, edad y nivel socioeconómico, evaluándose el nivel educacional y ocupacional del jefe de hogar (Dávila, Ormeño y Vera, 1998). El test está compuesto por 25 afirmaciones, las que se subdivide en dos escalas: Cuidado (12 ítem) y Sobreprotección (13 ítem); cada ítem se puntúa a través de una escala Likert en un rango de 0 a 3 puntos, quedando la escala de Cuidado con un puntaje máximo de 36 puntos y la de Sobreprotección con 39 puntos. El sujeto debe elegir aquella alternativa que mejor describa la relación con cada uno de sus padres, por separado; es decir, se contesta uno para Padre y otro para Madre, basado en los recuerdos hasta los 16 años, según criterio de los autores (Melis et al., 2001).

Como señalan Melis et al. (2001) cada escala puede ser utilizada de manera independiente o conjunta, obteniendo así un puntaje para Cuidado y otro para Sobreprotección. El obtener puntajes de ambas escalas permite elaborar cinco tipos de vínculos parentales: (a) Vínculo Óptimo, (b) Vínculo 
Tabla 1, Principales características de las muestras de agresores y no-agresores contra la pareja

\begin{tabular}{|c|c|c|c|c|c|}
\hline \multirow[b]{2}{*}{ Variables } & \multirow[b]{2}{*}{ Categorias } & \multicolumn{2}{|c|}{$\begin{array}{l}\text { Agresores contra } \\
\text { la Pareja }(n=32)\end{array}$} & \multicolumn{2}{|c|}{$\begin{array}{l}\text { No Agresores contra } \\
\text { la Pareja }(n=30)\end{array}$} \\
\hline & & $f$ & $\%$ & $f$ & $\%$ \\
\hline \multirow{6}{*}{$\begin{array}{l}\text { Convivencia } \\
\text { Diaria }\end{array}$} & Institución & 17 & $53.1 \%$ & 30 & $100 \%$ \\
\hline & Solo & 1 & $3.1 \%$ & & \\
\hline & Familia de origen & 4 & $12.5 \%$ & & \\
\hline & Solo con pareja & 2 & $6.3 \%$ & & \\
\hline & Pareja e hijos & 6 & $18.8 \%$ & & \\
\hline & Otras personas & 2 & $6.3 \%$ & & \\
\hline \multirow{4}{*}{$\begin{array}{l}\text { Situación } \\
\text { Laboral }\end{array}$} & Trabajo a tiempo completo & 13 & $40.6 \%$ & 10 & $33.3 \%$ \\
\hline & Trabajo media jornada & 4 & $12.5 \%$ & 4 & $13.3 \%$ \\
\hline & Trabajo ocasional & 5 & $15.6 \%$ & 3 & $10 \%$ \\
\hline & Cesante & 10 & $31.3 \%$ & 13 & $43.3 \%$ \\
\hline \multirow{5}{*}{$\begin{array}{l}\text { Estado } \\
\text { Civil }\end{array}$} & Soltero & 17 & $53.1 \%$ & 20 & $66.7 \%$ \\
\hline & Casado & 9 & $28.1 \%$ & 3 & $10 \%$ \\
\hline & Separado & 2 & $6.3 \%$ & 5 & $16.7 \%$ \\
\hline & Divorciado & 2 & $6.3 \%$ & 2 & $6.7 \%$ \\
\hline & Viudo & 2 & $6.3 \%$ & 0 & $0 \%$ \\
\hline \multirow{5}{*}{$\begin{array}{l}\text { Condición } \\
\text { Socioeconómica }\end{array}$} & Baja & 18 & $56.3 \%$ & 26 & $86.7 \%$ \\
\hline & Media Baja & 6 & $18.8 \%$ & 1 & $3.3 \%$ \\
\hline & Media & 4 & $12.5 \%$ & 1 & $3.3 \%$ \\
\hline & Media Alta & 3 & $9.4 \%$ & 1 & $3.3 \%$ \\
\hline & Alta & 1 & $3.1 \%$ & 1 & $3.3 \%$ \\
\hline \multirow{5}{*}{$\begin{array}{l}\text { Formación } \\
\text { académica }\end{array}$} & Básica Incompleta & 11 & $34.4 \%$ & 13 & $43.3 \%$ \\
\hline & Básica Completa & 4 & $12.5 \%$ & 2 & $6.7 \%$ \\
\hline & Media Incompleta & 9 & $28.1 \%$ & 4 & $13.3 \%$ \\
\hline & Media Completa & 7 & $21.9 \%$ & 9 & $30 \%$ \\
\hline & Superior Incompleta & 1 & $3.1 \%$ & 2 & $6.7 \%$ \\
\hline
\end{tabular}

Ausente o Débil, (c) Constricción Cariñosa, (d) Control Sin Afecto, (e) Promedio (Dávila, Ormeño y Vera, 1998).

Para efectos de esta investigación, y en concordancia con lo efectuado por Grimalt y Heresi (2012), se consideró como Tipo de apego inseguro los estilos parentales de Vínculo Ausente o Débil, Constricción Cariñosa y Control Sin Afecto; por su parte fue considerado como tipo de apego seguro el Vínculo Óptimo.

\section{Procedimiento}

Una vez contado con la autorización del Alcaide de Centro Penitenciario (CP) de Puerto Montt, y los respectivos jefes de las unidades jefa del Centro de Reinserción Social (CRS) se procedió a contactar a los internos y ejecutar la aplicación de instrumentos a los sujetos tanto de Reclusión Nocturna $(\mathrm{RN})$ como de régimen cerrado.

A todos los participantes se les entregó el consentimiento informado para su conocimiento del resguardo de la información obtenida y se les indicó las modalidades de entrevista. En primer lugar, se llevó a cabo la aplicación autoadministrada del instrumento EPQ-R y PBI. Luego, se dio paso a la entrevista para responder el protocolo de recogida de información y el manual SARA. El encuentro duró aproximadamente 90 minutos por sujeto.

Posteriormente, se analizaron los informes colaterales que incluyen, partes policiales, denuncias de víctimas, expedientes penitenciarios e informes psicológicos disponibles, para completar el SARA y así realizar una triangulación con la entrevista. 


\section{Análisis de datos}

En cuanto a las técnicas de análisis, en primer lugar, para determinar la confiabilidad, se usaron los coeficientes Kappa y Alfa de Cronbach. En segundo término, para demostrar los distintos tipos de validez, se trabajó con: (a) el coeficiente de correlación no-paramétrico de Spearman (ya que no hubo distribución normal en las variables); (b) la Curva Característica Operativa del Receptor (Curva ROC); (c) la Regresión Logística; y (d) la comparación de grupos a través de la U de Mann-Whitney (ya que no hubo distribución normal en las variable).

\section{Resultados}

Se presentan los resultados del estudio en base a los objetivos que se trazaron.

\section{Adaptación del instrumento}

El primer objetivo fue adaptar el instrumento, para ello se revisó la correspondencia entre los ítems originales en inglés, los de la versión española y la propuesta de cambios para la versión chilena. Luego, se seleccionaron 5 jueces expertos, a quienes se les facilitó, la definición operativa del constructo a evaluar y la batería de ítems adaptados junto con una pauta de evaluación en base a los 4 criterios sugeridos por Escobar-Pérez y Cuervo-Martínez (2008) que son suficiencia, claridad, relevancia y coherencia.

Las modificaciones resultantes de este procedimiento incluyeron la mantención del número de ítems, pero se precisó la redacción de algunos de ellos. Los cambios incluyen: (a) reducción de la cantidad de palabras (ítem 1); (b) reemplazo del concepto "violación" por el de "incumplimiento" (ítems 3,15 y 20); (c) operacionalización del concepto "reciente" en cual quedó en "durante el último año" (ítems 4, 5, 7, 8,

Tabla 2. Análisis de consistencia interna para el Manual SARA y sus dimensiones $(n=62)$

\begin{tabular}{|c|c|c|c|c|c|c|c|c|c|}
\hline Dimensión & Ítem & $\begin{array}{l}\text { Media } \\
(0-2)\end{array}$ & $\begin{array}{l}\text { Desviación } \\
\text { típica }\end{array}$ & $\begin{array}{l}\text { Correlación } \\
\text { ítem-total } \\
\text { Dimensión }\end{array}$ & $\begin{array}{l}\text { Correlación } \\
\text { ítem-total } \\
\text { Escala Total }\end{array}$ & $\begin{array}{c}\alpha \text { si se } \\
\text { elimina el } \\
\text { elemento } \\
\text { Dimensión }\end{array}$ & $\begin{array}{c}\alpha \text { si se elimina el } \\
\text { elemento Escala } \\
\text { Total }\end{array}$ & $\begin{array}{c}\alpha \\
\text { Dimensión }\end{array}$ & $\begin{array}{c}\alpha \\
\text { Escala } \\
\text { Total }\end{array}$ \\
\hline \multirow{3}{*}{$\begin{array}{l}\text { Historial } \\
\text { delictivo }\end{array}$} & 1 & 0.58 & 0.86 & 0.407 & 0.276 & 0.011 & 0.827 & \multirow{3}{*}{0.429} & \\
\hline & 2 & 1.13 & 0.914 & 0.299 & 0.246 & 0.257 & 0.83 & & \\
\hline & 3 & 0.23 & 0.584 & 0.096 & 0.394 & 0.523 & 0.821 & & \\
\hline \multirow{7}{*}{$\begin{array}{l}\text { Variables } \\
\text { psicosociales }\end{array}$} & 4 & 0.37 & 0.659 & 0.433 & 0.344 & 0.429 & 0.822 & \multirow{7}{*}{0.538} & \\
\hline & 5 & 0.58 & 0.759 & 0.144 & 0.015 & 0.556 & 0.839 & & \\
\hline & 6 & 0.84 & 0.909 & 0.224 & 0.259 & 0.535 & 0.829 & & \\
\hline & 7 & 0.31 & 0.667 & 0.384 & 0.292 & 0.449 & 0.825 & & \\
\hline & 8 & 0.24 & 0.619 & 0.43 & 0.107 & 0.435 & 0.832 & & \\
\hline & 9 & 0.11 & 0.367 & 0.224 & 0.081 & 0.52 & 0.83 & & \\
\hline & 10 & 0.18 & 0.497 & 0.117 & 0.483 & 0.546 & 0.818 & & \\
\hline \multirow{7}{*}{$\begin{array}{c}\text { Historial de } \\
\text { violencia } \\
\text { contra la } \\
\text { pareja }\end{array}$} & 11 & 0.68 & 0.845 & 0.729 & 0.692 & 0.758 & 0.803 & \multirow{7}{*}{0.816} & 0,827 \\
\hline & 12 & 0.19 & 0.568 & 0.413 & 0.327 & 0.813 & 0.823 & & \\
\hline & 13 & 0.32 & 0.719 & 0.61 & 0.571 & 0.783 & 0.812 & & \\
\hline & 14 & 0.52 & 0.805 & 0.601 & 0.573 & 0.784 & 0.811 & & \\
\hline & 15 & 0.39 & 0.754 & 0.444 & 0.48 & 0.811 & 0.816 & & \\
\hline & 16 & 0.5 & 0.741 & 0.583 & 0.517 & 0.787 & 0.814 & & \\
\hline & 17 & 0.52 & 0.695 & 0.508 & 0.494 & 0.8 & 0.816 & & \\
\hline \multirow{3}{*}{$\begin{array}{c}\text { Delito/ } \\
\text { Agresión } \\
\text { actual (Que } \\
\text { motiva la } \\
\text { valoración) }\end{array}$} & 18 & 0.72 & 0.818 & 0.694 & 0.718 & 0.411 & 0.802 & \multirow{3}{*}{0.712} & \\
\hline & 19 & 0.44 & 0.781 & 0.572 & 0.557 & 0.576 & 0.812 & & \\
\hline & 20 & 0.6 & 0.877 & 0.361 & 0.555 & 0.833 & 0.811 & & \\
\hline
\end{tabular}


9 y 14); (d) precisión del tipo de violencia física ejercida "contra la pareja o ex - pareja" y reemplazo del concepto "anterior" por "en el pasado" (ítems 11) y (e) complementación del concepto "amenazas de muerte creíbles" por “comprobación de amenazas" (ítems 13 y 19).

Posterior a ello, se aplicó el instrumento a 5 casos piloto para entrenar a los codificadores y evaluar la claridad de los ítems del protocolo. A partir de esta aplicación, no se generaron nuevos cambios. Con respecto a la codificación de los administradores del SARA, se buscó obtener un indicador de la consistencia de las puntuaciones asignadas, para ello se llevó a cabo un análisis de confiabilidad interobservadores usando el Kappa de margen fijo para múltiples observadores, cuyos valores pueden variar entre -1 y 1 , siendo el 0 un acuerdo inter-observadores posible de obtener sólo a través del azar mientras que el 0,7 sería el valor indicador de un acuerdo inter-observador aceptable (Randolph, 2008). Finalmente, el coeficiente Kappa obtenido para los cinco casos observados fue superior a 0,7 , permitiendo de esta manera ratificarse la consistencia de las puntuaciones entregadas por los codificadores.

\section{Confiabilidad}

Una vez aplicado el instrumento a la muestra definitiva, se realizó un análisis de la consistencia interna de los ítems del SARA, tanto para la escala total como para sus dimensiones, a través del coeficiente alfa de Cronbach. La fiabilidad de los ítems del manual SARA adaptado fue óptima para la escala total $(\alpha=827)$ y las dimensiones Historial de violencia contra la pareja $(\alpha=, 816)$ y Delito actual $(\alpha=, 712)$. Para las dimensiones Historial delictivo $(\alpha=, 429)$ y Variables psicosociales $(\alpha=, 538)$, la consistencia interna fue menor. Con respecto a la capacidad de discriminación (correlación ítem-total) de los ítems del SARA se observa que buena parte de los ítems tienen adecuados valores en las correlaciones elemento-total considerando la escala total como la dimensión a la que pertenecen (sobre 0,3 ). Sin embargo, existen ítems que correlacionan con el total del SARA de manera débil. Aun así, ante la posibilidad de eliminar estos ítems se visualiza que el valor alfa de
Cronbach no aumentaría de manera considerable, por lo que no se tomaron decisiones de eliminación de ítems en base a este criterio (ver Tabla 2).

\section{Validez Concurrente}

La primera hipótesis del estudio buscó correlacionar las puntuaciones del SARA adaptado con el puntaje de la escala $\mathrm{N}$ (Neuroticismo) del EPQ-R. Los resultados se dieron en el sentido esperado para la teoría, es decir, la correlación fue estadísticamente significativa, directa y baja $r h o=.290, p<$ .05. En términos generales, esto indica que los sujetos que puntúan alto en violencia contra la pareja según el SARA, tienden a puntuar alto en Neuroticismo. En consecuencia, la Hipótesis 1 tiene sustento empírico.

$\mathrm{Al}$ desglosar este resultado entre las dimensiones del manual SARA adaptado y la variable Neuroticismo es posible señalar que cada dimensión se correlaciona de manera diferente con la escala N del EPQ-R. Las dimensiones que presentaron una correlación estadísticamente significativa y directa fueron Historial delictivo rho $=, 299, p<, 05$ y Variables psicosociales rho $=, 350, p<, 01$. Cabe mencionar, que las dos dimensiones restantes (Delito actual e Historial de la violencia contra la pareja) carecen de correlación significativa con Neuroticismo (ver Tabla 3).

La segunda hipótesis buscó establecer diferencias entre la muestra clínica de agresores contra la pareja y la de no-agresores contra la pareja en el puntaje que entrega el SARA. El resultado indica que existen diferencias estadísticamente significativas entre ambas muestras en relación con la puntuación total del SARA adaptado $U=20, p<.01$, es decir, la muestra de agresores contra su pareja $M e=17$, $\mathrm{DT}=23.932$ puntúan significativamente más alto que la muestra de contraste de no-agresores contra la pareja $\mathrm{Me}$ $=3,5, \mathrm{DT}=3.391$, la magnitud de la diferencia fue grande $r=, 824$ (ver Tabla 4). Por ende, se cumple la Hipótesis 2 del estudio.

La hipótesis tres, intentó diferenciar las puntuaciones del SARA según el tipo de apego del participante. El resultado evidenció la existencia de diferencias estadísticamente significativas $U=259, p<, 01$, entre apego seguro $M e=7$,

Tabla 3. Matriz de correlaciones entre las puntuaciones del SARA y Neuroticismo

\begin{tabular}{lcccccc}
\hline $\begin{array}{l}\text { Validez } \\
\text { Concurrente }\end{array}$ & $\begin{array}{c}\text { SARA } \\
\text { total }\end{array}$ & $\begin{array}{c}\text { Historial } \\
\text { delictivo }\end{array}$ & $\begin{array}{c}\text { Variables } \\
\text { psicosociales }\end{array}$ & Historial de VCP & Delito actual \\
\hline Neuroticismo & rho & .290 & .299 & .350 & 0.192 & 0.091 \\
& $p$ & 0.022 & 0.018 & 0.005 & n.s. & n.s. \\
\hline
\end{tabular}


Tabla 4

Comparación de las medianas del Puntaje Total del SARA según tipo de muestra $(n=62)$ y tipo de apego $(n=60)$

\begin{tabular}{|c|c|c|c|c|c|c|c|c|}
\hline & $\mathrm{n}$ & Mediana & Rango Medio & DT & $U$ & $Z$ & $p$ & $r$ \\
\hline Agresores contra la pareja & 32 & 17.0 & 45.88 & 23.932 & \multirow{2}{*}{20} & \multirow{2}{*}{-6.488} & \multirow{2}{*}{0.000} & \multirow{2}{*}{0.824} \\
\hline No Agresores contra la pareja & 30 & 3.5 & 16.17 & 3.391 & & & & \\
\hline Apego seguro & 31 & 7 & 24.35 & 22.133 & \multirow{2}{*}{259} & \multirow{2}{*}{-2.821} & \multirow{2}{*}{0.005} & \multirow{2}{*}{0.364} \\
\hline Apego inseguro & 29 & 15 & 37.07 & 19.914 & & & & \\
\hline
\end{tabular}

$\mathrm{DT}=22.133$ y apego inseguro $M e=15, \mathrm{DT}=19.914$, en las puntuaciones totales del SARA adaptado. Lo cual da cuenta de que, los sujetos que poseen apego inseguro tienen puntuaciones significativamente más alta en el SARA que quienes tienen apego seguro, la magnitud de la diferencia fue moderada $r=, 364$. En consecuencia, este resultado apoya la Hipótesis 3 del estudio (ver Tabla 4).

\section{Validez Diagnóstica}

De manera complementaria, se buscó evaluar la capacidad que tienen los puntajes del SARA para predecir a través de un modelo de regresión si el individuo es agresor contra la pareja o no $(0=$ No agresores contra la pareja; $1=$ Agresores contra la pareja). El modelo resultó ser significativo $\left(\chi^{2}=\right.$ $63.554, \mathrm{p}<.01)$ y de excelente capacidad predictiva, un $93,5 \%$ a la hora de clasificar los sujetos. El riesgo relativo (odds ratio) de ser agresor fue de 1.862 cuando se tiene un puntaje alto en el SARA. Asimismo, por medio de un análisis de Curva ROC, se pretendió determinar la capacidad de clasificación de las puntuaciones SARA usando como criterio las mismas categorías $0=$ No agresores contra la pareja y $1=$ Agresores contra la pareja. El resultado sugiere una excelente capacidad del SARA para detectar los Verdadero Positivos (Sensibilidad $=, 938)$, es decir, los individuos que puntúan alto en el instrumento y que efectivamente son agresores contra la pareja (clasificados previamente). Por su parte, la capacidad del SARA para detectar a los individuos que no poseen la característica estudiada (Especificidad), es decir los sujetos no agresores contra la pareja (Verdadero Negativos), fue de ,933. El Área Bajo la Curva ROC fue de ,976, lo cual indica que es posible clasificar a los sujetos con una precisión del $97,6 \%$. Además, este análisis sugiere que el punto de corte a partir del cual el instrumento comienza a detectar a los agresores es de 8 puntos.

\section{Discusión}

Tal como se expuso anteriormente, el primer objetivo de este estudio se propuso adaptar los ítems del instrumento. Se logró generar un instrumento concordante con la versión en inglés y española, pero contextualizada al lenguaje propio de nuestra realidad nacional. Cabe destacar que para llegar a este resultado se siguieron las recomendaciones internacionales para adaptar, resguardar la validez de contenido del instrumento (juicio de expertos) y asegurar la comprensión de los ítems de parte de los evaluados y entrenamiento básico de los codificadores (aplicación piloto) (Balluerka, Gorostiaga, Alonso-Arbiol y Haranburu, 2007).

Por otro lado, respondiendo al segundo objetivo se obtuvieron adecuados indicadores de acuerdo interjueces y de consistencia interna global estimada a través del Alfa de Cronbach, por ende el instrumento arroja puntajes fiables. No obstante este resultado positivo, resulta necesario considerar que algunas dimensiones del instrumento presentan consistencia interna baja. Tal es el caso de Historial delictivo y variables psicosociales. Una explicación posible al respecto podría deberse al número de ítems de Historial delictivo el cual es reducido en comparación a las dimensiones restantes. Para variables psicosociales un argumento razonable podría deberse a las características específicas de este grupo de evaluado los cuales son internos. Por ejemplo, al evaluar el ítem 5 referente a problemas de ocupación o trabajo en el pasado en instancias de que la mayoría está recluida, lo que se traduce en respuestas disimiles al resto. A nivel estadístico esto se traduciría en una correlación ítem-total baja (ver Tabla 2).

El tercer objetivo buscó evaluar la validez concurrente del instrumento cuyos resultados sugieren que el SARA mide en una dirección concordante con las variables criterios Neuroticismo, tipo de muestra (agresor contra la pareja y no-agresor contra la pareja) y tipo de Apego.

La primera hipótesis en esta línea, comprobó la relación positiva entre Neuroticismo y puntuación en SARA, lo cual va en la dirección de estudios previos que confirman esta 
asociación. Los individuos con alto Neuroticismo tienden a presentar dificultades en la regulación de sus afectos y emociones, presentan inestabilidad emocional y afectivamente, con baja tolerancia a la frustración, inseguros, dependientes, conflictivos consigo mismo y con un alto nivel de ansiedad flotante aumentan su probabilidad de ser agresivos, debido a que en sus relaciones de pareja, se vuelven dependientes de sus relaciones, afectiva y emocionalmente (Castellano et al, 2004). De este modo, la inestabilidad afectiva como los celos atribuidos por la mujer a la pareja, presentarían una vinculación con la violencia física y emocional hacia las mujeres (Nóblega, 2012).

La segunda hipótesis comprobó que el SARA produce diferencias al comparar sujetos previamente clasificados como agresores contra la pareja con quienes no lo son.

En tercer lugar, se comprobó la hipótesis de diferencias estadísticamente significativas en las puntuaciones SARA adaptadas según el tipo de apego (seguro e inseguro) indicando una mayor puntuación del apego inseguro en el SARA. Todo esto confirma la evidencia empírica acumulada que respalda mayor vinculación del apego inseguro con violencia contra la pareja, incluso en lo referido a la transmisión intergeneracional de esta (Meloy, 2002). Es esperable que experiencias tempranas de violencia influyan, a su vez, en la violencia de pareja adulta a través del perjuicio que en cualquier relación genera el miedo al abandono y la evitación de la intimidad (Godbout et al., 2009). Incluso se plantea que las parejas de individuos de apego inseguro, tienen a su vez mayor probabilidad de tener apego inseguro, lo cual los inserta en una interacción que pudiera predisponer al riego de violencia. Por ejemplo Kessner y McKenry (1998) encontraron que los hombres agresores presentaban mayor apego temeroso, lo cual se manifestaba en que la rabia que actúa en los apegos seguros como una forma de protesta, en el apego temeroso se intensifica transformándose en conducta violenta que se incrementa en una escalada de agresividad. Por consiguiente este resultado aporta evidencia a la validez de la medición de violencia contra la pareja a través del SARA, puesto que mide en la dirección esperada.

Por último, al evaluar la validez diagnóstica del instrumento se encontraron excelentes resultados en la capacidad predictiva y de clasificación del SARA donde los porcentajes fueron superiores al $90 \%$ en ambos casos.

En cuanto a la relevancia metodológica de los hallazgos de este estudio, contar con un instrumento adaptado y con algunos indicadores de confiablidad y validez para la realidad nacional que evalúe de manera consistente el constructo violencia contra la pareja, llena un vacío psicométrico frente a una variable de alto impacto clínico y relevancia social.
Además de tener estimaciones más finas de prevalencia y, por ende, un mapeo más fidedigno de la problemática a nivel país (Rojas, 2013).

En cuanto a las implicancias prácticas de esta investigación, en primer lugar, los hallazgos referidos a la relación entre las puntuaciones del SARA y lo tipos de apego inseguro y Neuroticismo, permiten orientar el foco de la intervención psicoterapéutica con pacientes con tendencias violentas contra sus parejas, en aspectos clave, tales como la regulación de la inestabilidad emocional, en el sentido de que los potenciales agresores tienen dificultades para reconocer y, por consiguiente, regular las emociones como irritabilidad, impulsividad o ansiedad que estarían a la base de su comportamiento agresivo, cuando su pareja no cumple sus expectativas. Esto contribuiría a evitar la violencia-castigo que es la que se da en una relación de tipo complementaria (desigual), en donde las partes no tienen igual estatus (Ibaceta, 2011).

Por otro lado, el acompañamiento psicoterapéutico, también debiese tomar en cuenta la perspectiva de los vínculos pasados del agresor, y su injerencia en las relaciones presentes, considerando que la teoría señala que los agresores mantienen modelos internos operantes de apego desarrollados en el pasado y que se mantienen en las relaciones afectivas en el presente (Feeney y Noller, 2001) donde el otro (víctima) no es una figura de apego segura, por lo tanto no merecedora de confianza ni respeto.

En la misma línea de implicancias de los resultados del estudio, el contar con un instrumento que evalúe de forma consistente la violencia contra la pareja, permite a nivel de política social, prevenir futuras manifestaciones de esta condición, adelantando medidas de corte interventivo para el agresor como para su pareja (víctima), así como también, cuando corresponda, tomar medidas legales-protectoras que apunten a proteger la integridad de los demás miembros de la familia, puesto que es evidente que este fenómeno conlleva consecuencia en distintos planos para la familia en su totalidad.

En cuanto a las limitaciones del presente estudio, se puede nombrar que el tamaño muestral no permite generalizar estos resultados a todos los sujetos agresores contra la pareja. En segundo lugar, se puede establecer como una limitante que este estudio tiene una naturaleza transversal, por ende no permite establecer capacidad predictiva a partir de ello. Sólo se concluye que en esta muestra de individuos el SARA entrega puntuaciones consistentes, mide en la dirección esperada en base a otras variables y clasifica correctamente a los sujetos agresores contra la pareja. 
Respecto a las posibles líneas de investigación que surgen a partir de este trabajo se destaca la más inmediata y trascendental que sería el estudio de la validez predictiva del instrumento puesto que el SARA: fue diseñado para predecir el riesgo de violencia contra la pareja, por ende será un instrumento completo en cuanto a evidencia de validez y confiabilidad sólo cuando se haga un estudio con una muestra mayor, incluya un diseño longitudinal (seguimiento) para evaluar la reaparición de la conducta violenta contra la pareja a través del tiempo.

\section{Referencias}

Ainsworth, M. D. S., Blehar, M.C., Waters, E., y Wall, S. (1978). Patterns of attachment: A psychological study of the strange situation. Hillsdale, NJ: Erlbaum.

Andrés-Pueyo, A., y López, S. (2005). Manual para la valoración del riesgo de violencia contra la pareja. Barcelona: Ediciones de la Universidad de Barcelona.

Andrés-Pueyo, A., López, S., y Álvarez, E. (2008) Valoración del riesgo de violencia contra la pareja por medio del SARA. Papeles del Psicólogo, 29, pp.117-122.

Andrés-Pueyo,A. (2008). El riesgo de violencia, la delincuencia violenta y su gestión penitenciaria. En V.V.AA., V Jornadas de ATIP en Almagro. Madrid: ATIP.

Andrés-Pueyo, A. (2009). Parte I predicción del riesgo. Capítulo 1: La predicción de la violencia contra la pareja. En E. Echeberúa, J. Fernández-Montalvo y P. Corral. (Eds.), Predicción del riesgo de homicidio y de violencia grave en la relación de pareja. Instrumentos de evaluación del riesgo y adopción de medidas de protección. (21-49) España: Editorial Diseñarte.

Babcock, J. C., Jacobson, N. S., Gottman, J. M., y Yerington, T. P. (2000). Attachment, emotional regulation, and the function of marital violence: Differences between secure, preoccupied, and dismissing violent and non violent husbands. Journal of Family Violence, 15, 391-409.

Balluerka, N., Gorostiaga, A., Alonso-Arbiol, I., y Haranburu, M. (2007). La adaptación de instrumentos de medida de unas culturas a otras: una perspectiva práctica. Psicothema, 19, 124-133.

Bartholomew, K., y Horowiz, L. (1991) Attachment Styles Among Young Adults: A Test of a Four-Category Model. Journal of Personality and Social Psychology, 61, 226-244.

Bowlby, J. (1973). Separation: Anxiety y Anger. Attachment and Loss (vol. 2); (International psycho-analytical library $\mathrm{N}^{\circ}$ 95). London: Hogarth Press.

Buck, N., Leenaars, E., Emmelkamp, P., y Van Marle, H. (2012) Explaining the Relationship Between Insecure Attachment and Partner Abuse: The Role of Personality Characteristics. Journal of Interpersonal Violence, $X X, 1-22$.

Bustos, M. T., y Meneses, C. (1991). Adaptación del Eysenck Personality Questionnaire Revised Version a la población adulta del área metropolitana de Chile. (Tesis de Licenciatura en Psicología no publicada). Escuela de Psicología, Universidad Diego Portales.

Castellano, M., Lachica, E. Molina, A., y Villanueva, H. (2004). Violencia contra la mujer. El perfil del agresor: criterios de valoración del riesgo. Cuadernos de Medicina Forense, 35. Recuperado desde http://scielo. isciii.es/scielo.php?script=sci_arttextypid=S1135-76062004000100002

Carretero-Dios, H., y Pérez, C. (2005). Normas para el desarrollo y revisión de estudios instrumentales. International Journal of Clinical and Health Psychology, 5, 521-551.
Carabineros de Chile. (2013). Reporte estadístico Enero 2012/2013, nacional y regional. Recuperado desde http://www.carabineros.cl/ descarga/informe_estadistico_enero_2013.pdf

Cortés, M., Mestre, M., Tur, A., Samper, P., y Nácher, M. (Enero, 2007). Estilos de crianza en la adolescencia y su relación con el comportamiento prosocial. Revista Latinoamericana de Psicología, 39, Recuperado desde http://www.scielo.org.co/scielo.php?pid=S0120053420070002 00001 yscript=sci_arttext

Dávila, M., Ormeño, V., y Vera, V. (1998). Estandarización del P.B.I: (Parental Bonding Instrument): versión adaptada a la población entre 16 y 64 años del Gran Santiago. (Tesis para optar al grado de licenciado en Psicología), Universidad Diego Portales.

Escobar-Pérez, J., y Cuervo-Martínez, A. (2008). Validez de contenido y juicio de expertos: una aproximación a su utilización. Avances en Medición, 6, 27-36.

Eysenck, H. (1982). Fundamentos biológicos de la personalidad. Barcelona: Fontanella.

Feeney, J., y Noller, P. (2001). Apego adulto. Bilbao: Editorial Desclée de Brouwer.

Fraley, C., y Shaver, Ph. (2000) Adult Romantic Attachment: Theoretical Developments, Emerging Controversies and Unanswered Questions. Review of General Psychology, 4, 132-154.

Gempp, R., y Chesta, S. (2007). Errores Estándar de Medida Condicionales para las normas metropolitanas de la adaptación chilena del EPQ-R: Aplicación de un modelo binomial a un test de personalidad. Terapia Psicológica, 25, 51-62.

Godbout, N., Dutton, D., Lussier, Y., y Sabourin, S. (2009). Early exposure to violence, domestic violence, attachment representations, and marital adjustment. Personal Relationships, 16, 365-384.

Grimalt, L., y Heresi, E. (2012). Estilos de apego y representaciones maternas durante el embarazo. Revista Chilena Pediatría, 83, 239-246.

Holtzworth-Munroe, A., Meehan, J. C., Herron, K., Rehman, U., y Stuart, G. L. (2003). Do subtypes of maritally violent men continue to differ over time?. Journal of Consulting and Clinical Psychology, $71,728-740$

Ibaceta, F. (2004). Hombres que ejercen violencia en la pareja: reflexiones de una experiencia de atención individual. Terapia Psicológica, 22, 157-164.

Ibaceta, F. (2011). Violencia en la Pareja: ¿Es Posible la Terapia Conjunta?. Terapia Psicológica, 29, 117-125.

Ibañez, M. (1997). Primeros datos de la versión en castellano del cuestionario revisado de personalidad de Eysenck (EPQ-R). Recuperado desde http://www.uji.es/bin/publ/edicions/jfil/ eysenck.pdf

Kaplan, M., y Liberman, L. (1992). Estandarización del Test EPQ-R (Eysenck Personality Questionnaire, Revised version) adaptación BustosMeneses 1991, a la población urbana adulta del área metropolitana de Chile. (Tesis de Licenciatura en Psicología no publicada, Escuela de Psicología), Universidad Diego Portales.

Kesner, J., y McKenry, P. (1998). The role of childhood attachment factors in predicting male violence toward female intimates. Journal of Family Violence, 13, 417-432.

Kropp, P. R., Hart, S. D., Webster, C. D., y Eaves, D. (1994). Manual for the Spousal Assault Risk Assessment Guide. Vancouver, BC: British Columbia Institute on Family Violence.

Kropp, P. R., Hart, S. D., Webster, C. W., y Eaves, D. (1995). Manual for the Spousal Assault Risk Assessment Guide, 2nd ed. Vancouver, BC: British Columbia Institute on Family Violence.

Kropp, P. R., Hart, S. D., Webster, C. W., y Eaves, D. (1998). Spousal Assault Risk Assessment: User's Guide. Toronto: Multi-Health Systems, Inc.

Kropp, P., y Hart, S. (2000). The Spousal Assault Risk Assessment (SARA) Guide: Reliability and Validity in Adult Male Offenders. Law and Human Behavior, 1, pp. 101-108.

Loinaz, I., y Echeburúa, E. (2012). Apego adulto en agresores de pareja. Revista Acción psicológica, 9, 33-46. Recuperado desde http://www. ehu.es/echeburua/pdfs/ Apego\%20adulto \%20en\%20agresores\%20 de\%20pareja.pdf 
López, S., y Andrés-Pueyo, A. (2007). Adaptación de la SARA Evaluación del riesgo de violencia de pareja. Àmbit social $i$ criminològic. Departament de Justícia. Recuperado desde http://www20.gencat.cat/ docs/Justicia/Documents/ARXIUS/SC-3-139-07-cas.pdf

Melis, F., Dávila, M., Ormeño, V., Vera, V., Greppi, C., y Gloger, S. (2001). Estandarización del P.B.I. (Parental Bonding Instrument), versión adaptada a la población entre 16 y 64 años del Gran Santiago. Revista chilena de neuro-psiquiatría, 39, 132-139. Recuperado desde http://www.scielo.cl/scielo.php?script=sci_arttextypid=S0717$92272001000200005 \mathrm{ylng}=$ enytlng=es . doi: $10.4067 / \mathrm{S} 0717$ 92272001000200005

Meloy, J. R. (2002) Pathologies of Attachment, Violence, and Criminality. En A. Goldstein, ed., Handbook of Psychology, Vol II. New York: Wiley.

Montero, I., y León, O.G. (2007). A guide for naming research studies in Psychology. International Journal of Clinical and Health Psychology, 7, 847-862.
Muñiz, J., Elousa, P., y Hambleton, R. K. (2013). Directrices para la traducción y adaptación de los tests: segunda edición. Psicothema, 25, 151-157. doi: 10.7334/psicothema2013.2

Nóblega, M. (2012). Características de los agresores en la violencia hacia la pareja. Liberabit. Revista de Psicología, 18, 59-67. Recuperado desde http://www.redalyc.org/articulo.oa? id $=68623931008$

Randolph, J. (2008). Online Kappa Calculator. Recuperado desde http:// justus.randolph.name/kappa.

Rojas, S. (2013). Femicidio y feminicidio en Latinoamérica. En Boll, H. (Ed.) Feminicidio un fenómeno global de Madrid a Santiago. Unión Europea.

Servicio Nacional de la Mujer. (2010). Violencia Intrafamiliar en Cifras. Recuperado desde http://sernam.cl/portal/images/documentos/campania_maltrato2010/vif_cifras.pdf 


\section{Anexos}

\section{Anexo 1}

\section{Manual para la valoración del riesgo de violencia contra la pareja (SARA) versión chilena}

Hoja de Codificación

\begin{tabular}{|l|l|}
\hline Identificación del Evaluado(a): & $\begin{array}{l}\text { Fecha: / / } \\
\text { Firma: }\end{array}$ \\
\hline Nombre del Evaluador(a): & Firma: \\
\hline
\end{tabular}

\begin{tabular}{|c|c|c|c|c|}
\hline $\begin{array}{c}\text { Dimensión I: Historial } \\
\text { Delictivo }\end{array}$ & & $\begin{array}{c}\text { Valoración } \\
(0-1-2)\end{array}$ & & $\begin{array}{c}\text { Ítem Crítico } \\
(\mathbf{0} \text { o } 1)\end{array}$ \\
\hline $\begin{array}{l}\text { 1. Violencia anterior contra } \\
\text { familiares }\end{array}$ & $\begin{array}{c}\text { No existe evidencia de } \\
\text { agresión física, ni de in- } \\
\text { tentos reales o amenazas } \\
\text { de agresiones } \\
(0)\end{array}$ & $\begin{array}{l}\text { Amenaza de agresión } \\
\text { física a un miembro de la } \\
\text { familia en el pasado } \\
\text { (1) }\end{array}$ & $\begin{array}{l}\text { Agresión física o intento } \\
\text { real de agresión a un } \\
\text { miembro de la familia en } \\
\text { el pasado } \\
\text { (2) }\end{array}$ & \\
\hline $\begin{array}{l}\text { 2. Violencia anterior contra } \\
\text { desconocidos o conocidos } \\
\text { no- familiares }\end{array}$ & $\begin{array}{c}\text { No existe evidencia de } \\
\text { agresiones físicas ni de } \\
\text { intentos reales o ame- } \\
\text { nazas a conocidos (no } \\
\text { familiares) o desconoci- } \\
\text { dos en el pasado } \\
(0)\end{array}$ & $\begin{array}{l}\text { Existe evidencia de ame- } \\
\text { nazas de agresión física a } \\
\text { persona conocidas (no fa- } \\
\text { miliares) o desconocidos } \\
\text { (1) }\end{array}$ & $\begin{array}{c}\text { Existe evidencia de agre- } \\
\text { sión física o intento real } \\
\text { de agresión a personas } \\
\text { conocidas (no familiares) } \\
\text { o desconocidos en el } \\
\text { pasado } \\
\text { (2) }\end{array}$ & \\
\hline $\begin{array}{l}\text { 3. Incumplimiento de la } \\
\text { libertad condicional u otras } \\
\text { medidas judiciales similares }\end{array}$ & $\begin{array}{l}\text { No existe evidencia his- } \\
\text { tórica de incumplimiento } \\
\text { de medidas judiciales } \\
\text { dictadas por Tribunales } \\
(0)\end{array}$ & $\begin{array}{c}\text { Existe evidencia del } \\
\text { incumplimiento de la li- } \\
\text { bertad condicional u otras } \\
\text { medidas judiciales que no } \\
\text { han producido condenas o } \\
\text { arrestos } \\
\text { (1) }\end{array}$ & $\begin{array}{l}\text { Existe evidencia de con- } \\
\text { denas o arrestos por violar } \\
\text { la libertad condicional u } \\
\text { otras medida judiciales } \\
\text { (2) }\end{array}$ & \\
\hline
\end{tabular}

\begin{tabular}{|c|c|c|c|c|}
\hline $\begin{array}{c}\text { Dimensión II: Variables } \\
\text { Psicosociales }\end{array}$ & \multicolumn{3}{|c|}{$\begin{array}{l}\text { Valoración } \\
(0-1-2)\end{array}$} & $\begin{array}{l}\text { Ítem Crítico } \\
\quad\left(\begin{array}{l}\mathbf{0} \\
\text { o }\end{array} \mathbf{1}\right)\end{array}$ \\
\hline $\begin{array}{l}\text { 4. Problemas en la relación } \\
\text { de pareja durante el último } \\
\text { año }\end{array}$ & $\begin{array}{l}\text { No hay cambio en el } \\
\text { estado de la relación } \\
\text { de pareja a lo largo del } \\
\text { último año } \\
\text { (0) }\end{array}$ & $\begin{array}{l}\text { Conflicto de pareja que no } \\
\text { ha sido causal de separa- } \\
\text { ción y/o distanciamiento a } \\
\text { lo largo del último año. } \\
\text { (1) }\end{array}$ & $\begin{array}{l}\text { Conflicto de pareja causal } \\
\text { de separación y/o distan- } \\
\text { ciamiento a lo largo del } \\
\text { último año } \\
\text { (2) }\end{array}$ & \\
\hline $\begin{array}{l}\text { 5. Problemas de ocupación/ } \\
\text { trabajo durante el último } \\
\text { año }\end{array}$ & $\begin{array}{l}\text { Actualmente en situación } \\
\text { de empleo y con una } \\
\text { historia laboral estable a } \\
\text { lo largo del último año } \\
\text { (0) }\end{array}$ & $\begin{array}{l}\text { Actualmente en situación } \\
\text { de desempleo, pero con } \\
\text { una historia laboral esta- } \\
\text { ble a lo largo del último } \\
\text { año } \\
\text { (1) }\end{array}$ & $\begin{array}{l}\text { Actualmente en situación } \\
\text { de desempleo y con una } \\
\text { historia laboral inestable a } \\
\text { lo largo del último año } \\
\text { (2) }\end{array}$ & \\
\hline
\end{tabular}




\begin{tabular}{|c|c|c|c|c|}
\hline $\begin{array}{l}\text { 6. Víctima y/o testigo de } \\
\text { violencia familiar en la } \\
\text { infancia y/o adolescencia }\end{array}$ & $\begin{array}{c}\text { No existe evidencia de } \\
\text { que el individuo haya } \\
\text { sido víctima ni testigo } \\
\text { de violencia familiar } \\
\text { durante la adolescencia } \\
\text { y/o infancia } \\
(0)\end{array}$ & $\begin{array}{c}\text { Existe evidencia de que } \\
\text { el individuo haya sido } \\
\text { víctima y/o testigo de vio- } \\
\text { lencia familiar infrecuente } \\
\text { o leve durante la adoles- } \\
\text { cencia y/o infancia } \\
\text { (1) }\end{array}$ & $\begin{array}{c}\text { Existe evidencia de que } \\
\text { el individuo haya sido } \\
\text { víctima y/o testigo de vio- } \\
\text { lencia familiar frecuente o } \\
\text { grave durante la adoles- } \\
\text { cencia y/o infancia } \\
\text { (2) }\end{array}$ & \\
\hline $\begin{array}{l}\text { 7. Consumo/Abuso de } \\
\text { drogas y/o alcohol durante } \\
\text { el último año }\end{array}$ & $\begin{array}{l}\text { No existe evidencia de } \\
\text { que el individuo sea } \\
\text { consumidor ni adicto a } \\
\text { drogas y/o alcohol a lo } \\
\text { largo del último año } \\
(0)\end{array}$ & $\begin{array}{c}\text { Existe evidencia de } \\
\text { consumo leve o moderado } \\
\text { de drogas y/o alcohol a lo } \\
\text { largo del último año } \\
\text { (1) }\end{array}$ & $\begin{array}{l}\text { Existe evidencia de consu- } \\
\text { mo ó adicción grave de } \\
\text { drogas y/o alcohol a lo } \\
\text { largo del último año } \\
\text { (2) }\end{array}$ & \\
\hline $\begin{array}{l}\text { 8. Ideas/Intentos de suicido } \\
\text { y/o homicidio durante el } \\
\text { último año }\end{array}$ & $\begin{array}{c}\text { No existe evidencia de } \\
\text { que el individuo a lo } \\
\text { largo del último año haya } \\
\text { tenido ideas o realizado } \\
\text { intentos de suicidio y/o } \\
\text { homicidio } \\
(0)\end{array}$ & $\begin{array}{c}\text { Existe evidencia de } \\
\text { ideación suicida o fantasía } \\
\text { homicida a lo largo del } \\
\text { último año } \\
\text { (1) }\end{array}$ & $\begin{array}{c}\text { Existe evidencia de inten- } \\
\text { to grave/real de suicidio } \\
\text { u homicidio a lo largo del } \\
\text { último año } \\
\text { (2) }\end{array}$ & \\
\hline $\begin{array}{l}\text { 9. Síntomas psicóticos y/o } \\
\text { síntomas maníacos durante } \\
\text { el último año }\end{array}$ & $\begin{array}{c}\text { No existe evidencia de } \\
\text { síntomas de tipo psicóti- } \\
\text { co o maniático a lo largo } \\
\text { del último año } \\
(0)\end{array}$ & $\begin{array}{c}\text { Existe evidencia de } \\
\text { síntomas ligeros y/o mo- } \\
\text { derados de tipo psicótico } \\
\text { o maniático a lo largo del } \\
\text { último año } \\
\text { (1) }\end{array}$ & $\begin{array}{c}\text { Existe evidencia de } \\
\text { síntomas graves y/o } \\
\text { intensos de tipo psicótico } \\
\text { o maniático a lo largo del } \\
\text { último año } \\
\text { (2) }\end{array}$ & \\
\hline $\begin{array}{l}\text { 10. Trastorno de persona- } \\
\text { lidad con ira, impulsividad } \\
\text { y/o inestabilidad conductual }\end{array}$ & $\begin{array}{c}\text { No existe evidencia de } \\
\text { presencia de un trastorno } \\
\text { de personalidad caracte- } \\
\text { rizado por la presencia de } \\
\text { problemas de ira, impul- } \\
\text { sividad y/o inestabilidad } \\
\text { conductual } \\
(0)\end{array}$ & $\begin{array}{c}\text { Existe evidencia de } \\
\text { trastorno de personali- } \\
\text { dad caracterizado por la } \\
\text { presencia de problemas } \\
\text { leves o moderados de ira, } \\
\text { impulsividad y/o inestabi- } \\
\text { lidad conductual } \\
(1)\end{array}$ & $\begin{array}{c}\text { Existe evidencia de } \\
\text { trastorno de personali- } \\
\text { dad caracterizado por } \\
\text { la presencia de graves } \\
\text { problemas de ira, impul- } \\
\text { sividad y/o inestabilidad } \\
\text { conductual } \\
\text { (2) }\end{array}$ & \\
\hline $\begin{array}{l}\text { Dimensión III: Historial de } \\
\text { violencia contra la pareja. }\end{array}$ & \multicolumn{3}{|c|}{$\begin{array}{c}\text { Valoración } \\
(0-1-2)\end{array}$} & $\begin{array}{l}\text { Ítem Crítico } \\
(\mathbf{0} \text { o 1) }\end{array}$ \\
\hline $\begin{array}{c}\text { 11. Violencia física contra } \\
\text { la pareja o ex-pareja en el } \\
\text { pasado }\end{array}$ & $\begin{array}{c}\text { No existe evidencia } \\
\text { de que en el pasado se } \\
\text { hubiese agredido verbal } \\
\text { ni físicamente a su pareja } \\
\text { actual (o anteriores) } \\
(0)\end{array}$ & $\begin{array}{l}\text { Existe evidencia de agre- } \\
\text { siones verbales o intentos } \\
\text { de agresiones físicas en } \\
\text { el pasado contra la pareja } \\
\text { actual (o anteriores) } \\
(1)\end{array}$ & $\begin{array}{c}\text { Existe evidencia de agre- } \\
\text { siones físicas en el pasado } \\
\text { contra la pareja actual (o } \\
\text { anteriores) } \\
\text { (2) }\end{array}$ & \\
\hline $\begin{array}{l}\text { 12: Violencia sexual y/o } \\
\text { ataques de celos en el } \\
\text { pasado }\end{array}$ & $\begin{array}{c}\text { No existe evidencia } \\
\text { de agresiones sexua- } \\
\text { les actuales o pasadas } \\
\text { sobre la pareja actual (o } \\
\text { anteriores) y tampoco } \\
\text { historial de graves celos } \\
\text { sexuales }(0)\end{array}$ & $\begin{array}{c}\text { Existe intento de agresión } \\
\text { sexual en el pasado de } \\
\text { su pareja sentimental o } \\
\text { existencia de un historial } \\
\text { de graves celos sexuales } \\
\text { (1) }\end{array}$ & $\begin{array}{c}\text { Existe agresión sexual } \\
\text { en el pasado de su pareja } \\
\text { sentimental o, al menos } \\
\text { un acto de agresión física } \\
\text { en el contexto de un grave } \\
\text { ataque de celos de natura- } \\
\text { leza sexual (2) }\end{array}$ & \\
\hline $\begin{array}{l}\text { 13. Uso de armas y/o com- } \\
\text { probación de amenazas de } \\
\text { muerte en el pasado }\end{array}$ & $\begin{array}{c}\text { No existe evidencia de } \\
\text { amenazas de muerte, } \\
\text { lesiones graves o ataques } \\
\text { con armas hacia su pare- } \\
\text { ja actual (o anteriores) } \\
(0)\end{array}$ & $\begin{array}{c}\text { Existe evidencia de ame- } \\
\text { nazas de utilizar armas } \\
\text { en el futuro o amenazas } \\
\text { de lesiones graves en el } \\
\text { pasado } \\
\text { (1) }\end{array}$ & $\begin{array}{c}\text { Existe evidencia de } \\
\text { amenazas creíbles muerte } \\
\text { o uso de armas con esa } \\
\text { intención en el pasado } \\
\text { (2) }\end{array}$ & \\
\hline
\end{tabular}




\begin{tabular}{|c|c|c|c|}
\hline $\begin{array}{c}\text { 14. Incremento en la fre- } \\
\text { cuencia o gravedad de las } \\
\text { agresiones en el último año }\end{array}$ & $\begin{array}{l}\text { No existe evidencia del } \\
\text { aumento de la frecuen- } \\
\text { cia y/o gravedad de las } \\
\text { conductas agresivas a lo } \\
\text { largo del último año } \\
(0)\end{array}$ & $\begin{array}{c}\text { Existe evidencia de un } \\
\text { posible incremento en la } \\
\text { frecuencia y/o gravedad } \\
\text { de las conductas agresivas } \\
\text { a lo largo del último año } \\
\text { (1) }\end{array}$ & $\begin{array}{c}\text { Existe evidencia de un } \\
\text { indudable aumento en la } \\
\text { frecuencia o gravedad de } \\
\text { las conductas agresivas a } \\
\text { lo largo del último año } \\
\text { (2) }\end{array}$ \\
\hline $\begin{array}{l}\text { 15. Incumplimiento de las } \\
\text { órdenes de alejamiento en } \\
\text { el pasado }\end{array}$ & $\begin{array}{l}\text { No existe evidencia de } \\
\text { que en el pasado haya } \\
\text { incumplido las medidas } \\
\text { de protección dictadas } \\
\text { Tribunales } \\
(0)\end{array}$ & $\begin{array}{c}\text { Existe evidencia de } \\
\text { transgresión(es) de las } \\
\text { medidas de protección } \\
\text { dictadas por Tribunales, } \\
\text { las que no finalizaron con } \\
\text { arresto o detención } \\
\text { (1) }\end{array}$ & $\begin{array}{l}\text { Existe evidencia de arresto } \\
\text { o detención anterior por } \\
\text { haber incumplido las } \\
\text { medidas de protección } \\
\text { dictadas por Tribunales } \\
\text { (2) }\end{array}$ \\
\hline $\begin{array}{c}\text { 16. Minimización o } \\
\text { negación de la violencia } \\
\text { cometida hacia la pareja en } \\
\text { el pasado }\end{array}$ & $\begin{array}{l}\text { No existen discrepancias } \\
\text { con la documentación } \\
\text { que certifica sus actos } \\
\text { agresivos anteriores } \\
(0)\end{array}$ & $\begin{array}{c}\text { Minimización relativa o } \\
\text { negación de haber realiza- } \\
\text { do en el pasado conductas } \\
\text { agresivas a pesar de la } \\
\text { evidencia documentada, } \\
\text { por ejemplo: niega algu- } \\
\text { nas de las agresiones pero } \\
\text { admite otras } \\
\text { (1) }\end{array}$ & $\begin{array}{c}\text { Minimización extrema o } \\
\text { negación de haber realiza- } \\
\text { do en el pasado conductas } \\
\text { agresivas a pesar de la } \\
\text { evidencia documentada, } \\
\text { por ejemplo: niega la } \\
\text { responsabilidad personal } \\
\text { en la mayoría o todos los } \\
\text { actos agresivos anteriores } \\
(2)\end{array}$ \\
\hline $\begin{array}{l}\text { 17. Actitudes que apoyan, } \\
\text { justifican o consienten la } \\
\text { violencia contra la pareja }\end{array}$ & $\begin{array}{l}\text { No existe evidencia de } \\
\text { que el individuo tenga } \\
\text { actitudes que apoyen, } \\
\text { justifiquen o consientan } \\
\text { la violencia de pareja } \\
\text { (0) }\end{array}$ & $\begin{array}{c}\text { Existe evidencia de } \\
\text { aparentes actitudes que } \\
\text { apoyan, justifican o } \\
\text { consienten la violencia de } \\
\text { pareja } \\
\text { (1) }\end{array}$ & $\begin{array}{c}\text { Existe evidencia de la } \\
\text { mantención de actitudes } \\
\text { que apoyan, justifican o } \\
\text { consienten la violencia de } \\
\text { pareja (2) }\end{array}$ \\
\hline
\end{tabular}

\begin{tabular}{|c|c|c|c|c|}
\hline $\begin{array}{l}\text { Dimensión IV: Delito/ } \\
\text { Agresión Actual } \\
\text { (Oue motiva la valoración) }\end{array}$ & \multicolumn{3}{|c|}{$\begin{array}{c}\text { Valoración } \\
(0-1-2)\end{array}$} & $\begin{array}{c}\text { Ítem Crítico } \\
(\mathbf{0} \text { o } \mathbf{1})\end{array}$ \\
\hline $\begin{array}{l}\text { 18. Violencia física y/o } \\
\text { sexual grave }\end{array}$ & $\begin{array}{c}\text { No existe evidencia } \\
\text { de violencia física ni } \\
\text { sexual, en la acción que } \\
\text { se valora } \\
(0)\end{array}$ & $\begin{array}{c}\text { Existe evidencia de } \\
\text { violencia moderada o leve } \\
\text { en la acción violenta y } \\
\text { delictiva que se valora } \\
\text { (1) }\end{array}$ & $\begin{array}{c}\text { Existe evidencia de vio- } \\
\text { lencia severa, grave o se- } \\
\text { xual en la acción violenta } \\
\text { y delictiva que se valora } \\
\text { (2) }\end{array}$ & \\
\hline $\begin{array}{l}\text { 19. Uso de armas y/o com- } \\
\text { probación de amenazas de } \\
\text { muerte }\end{array}$ & $\begin{array}{l}\text { No hay evidencia de: } \\
\text { a) el uso de armas o de } \\
\text { amenazas futuras de } \\
\text { utilizarlas y b) amenazas } \\
\text { creíbles de muerte o de } \\
\text { lesiones graves en la } \\
\text { acción violenta que se } \\
\text { valora } \\
(0)\end{array}$ & $\begin{array}{c}\text { Existe evidencia de } \\
\text { amenazas de utilización } \\
\text { de armas o amenazas } \\
\text { creíbles de lesiones físicas } \\
\text { importantes en la acción } \\
\text { violenta que se valora } \\
\text { (1) }\end{array}$ & $\begin{array}{c}\text { Existe evidencia de la } \\
\text { utilización de armas o } \\
\text { intentos de asesinato creí- } \\
\text { bles en la acción violenta } \\
\text { que se valora } \\
\text { (2) }\end{array}$ & \\
\hline $\begin{array}{l}\text { 20. Incumplimiento de las } \\
\text { órdenes de alejamiento }\end{array}$ & $\begin{array}{l}\text { La acción violenta que } \\
\text { justifica la valoración } \\
\text { no se ha realizado bajo } \\
\text { la orden de "alejamien- } \\
\text { to" que ha impuesto } \\
\text { Tribunales o bien el in- } \\
\text { dividuo nunca tuvo esta } \\
\text { orden por cumplir } \\
(0)\end{array}$ & $\begin{array}{l}\text { La acción violenta que } \\
\text { justifica la valoración se } \\
\text { ha realizado bajo la orden } \\
\text { de "alejamiento" que ha } \\
\text { impuesto Tribunales, la } \\
\text { que no ha sido causal de } \\
\text { detención o arresto } \\
\text { (1) }\end{array}$ & $\begin{array}{l}\text { La acción violenta que } \\
\text { justifica la valoración } \\
\text { se ha realizado bajo la } \\
\text { petición de Tribunales por } \\
\text { haber incumplido la orden } \\
\text { de "alejamiento" impuesta } \\
\text { por Tribunales } \\
\text { (2) }\end{array}$ & \\
\hline
\end{tabular}

